\title{
Adequação de modelo compartimental para exames de tomografia por emissão de pósitrons
}

\author{
João Eduardo Maeda Moreira da Silva*, Sérgio Shiguemi Furuie
}

Resumo O presente trabalho objetiva a seleção do modelo compartimental mais adequado ao estudo da dinâmica fisiológica em exames de tomografia por emissão de pósitrons (PET). Para tanto, propõe-se utilizar o critério de informação de Akaike para a seleção do modelo, e a metodologia de equações de sensibilidade e o algoritmo de Levenberg-Marquardt para a tarefa de estimação de parâmetros característicos e, consequentemente, do erro residual da estimativa. São consideradas três estruturas compartimentais compostas, respectivamente, por dois compartimentos e duas constantes características, três compartimentos e quatro constantes características e quatro compartimentos e seis constantes características. Os dados considerados neste trabalho foram sintetizados preocupando-se em reunir as principais características de um exame de tomografia real, tais como tipo e nível de ruído e morfologia de função de excitação do sistema. Aplicando-se a metodologia proposta em três níveis de ruído (baixo, médio e alto), obteve-se concordância do melhor modelo em graus forte e considerável (com índices de Kappa iguais a 0,95, 0,93 e 0,63, respectivamente). Observou-se que, com elevado nível de ruído e modelos mais complexos (quatro compartimentos), a classificação se deteriora devido ao pequeno número de dados para a decisão. Foram desenvolvidos programas e uma interface gráfica que podem ser utilizados na investigação, elaboração, simulação, estimativa de parâmetros e identificação do modelo compartimental para apoio e análise de diagnósticos clínicos e práticas científicas.

Palavras-chave Seleção de modelos compartimentais, Tomografia por Emissão de Pósitrons (PET), Modelagem.

\section{Adequacy of compartmental model for positron emission tomography examinations}

Abstract The objective of this work is the determination of the most adequate compartmental model for the study
of physiological dynamics based on positron emission tomography (PET) exams. We propose the use of
Akaike's information criterion for the optimal model selection, and Levenberg-Marquardt algorithm with
sensitivity equations for the task of estimating the characteristic parameters of the differential equations
describing the models. We have considered three compartmental structures represented, respectively, by two
compartments and two characteristic constants, three compartments and four characteristic constants and
four compartments and six characteristics constants. The data considered in this work were synthesized taking
into account key features of a real tomography exam, such as type and level of noise and morphology of the
input function of the system. Applying the proposed methodology with three noise levels (low, medium and
high), we obtained agreement of the best model with strong and considerable degrees (with Kappa indexes
equal to 0.95, 0.93 and 0.63, respectively). It was observed that, with high noise level and more complex
models (four compartments), the classification is deteriorated due to lack of data for the decision. Programs
have been developed and a graphical interface that can be used in research, development, simulation and
parameter identification of compartmental models, supporting analysis of clinical diagnostics and scientific
practices.

Keywords Compartimental model selection, Positron Emission Tomography (PET), Modeling. 


\section{Extended Abstract}

\section{Introduction}

Some heart disease detection and myocardium feasibility analysis can be performed based on estimation of cardiac dynamics via mathematical modeling (Carson, 2005). Positron emission tomography (PET) exams allow the use of well-known compartmental models in the study of glucose metabolism, by considering the variation over time of the concentration of a tracer substance. The procedure of interest using PET involves the following steps (Figure 1): a) initially, injection of a tracer into radial artery of the subject; b) radioactive counts and reconstruction are carried out by PET, that provides a series of images showing the concentration of the tracer as a function of time (Huang et al., 1980); c) the regions of interest (ROI) are delimited by image processing. The objective of this work is the determination of the most adequate compartmental model for the study of physiological dynamics based on positron emission tomographic (PET) exams.

\section{Material and Methods}

We propose the use of Akaike's information criterion for the optimal model selection, and Levenberg-Marquardt algorithm with sensitivity equations (Figure 3) for the task of estimating the characteristic parameters of the differential equations describing the models. We have considered three compartmental structures represented, respectively, by two compartments and two characteristic constants, three compartments and four characteristic constants and four compartments and six characteristics constants (Figure 2). The data considered in this work were synthesized taking into account key features of a real tomographic exam, such as type and level of noise and morphology of the input function of the system.

\section{Results}

The proposed approach was applied to nine configurations of simulated experiments: three compartimental models and three levels of noise. Table 1 shows detailed results for two compartments model and medium noise. Other results are in (Maeda, 2010). The first column indicates the experiment (instance of noise), the two next columns ( $K_{1}$ and $k_{2}$ ) show the actual values of the constants to be estimated, and the following columns show the estimated values for the constants and Akaike index for each model, and finally, in the last column, the model suggested by the proposed approach. Tables 2-4 summarize the results considering the three noise levels (low, medium and high). We obtained agreement of the best model with strong and considerable degrees with Kappa indexes equal to 0.95, 0.93 and 0.63 , respectively. It was observed that, with high noise level and more complex models (four compartments), the classification is deteriorated due to lack of data for the decision. Programs have been developed and a graphical interface that can be used in research, development, simulation and parameter identification of compartmental models, supporting analysis of clinical diagnostics and scientific practices.

\section{Discussion and Conclusion}

This work aimed at selection of adequate models based on Akaike's information criterion. To this end we also investigated all relevant stages for compartmental analysis, in particular the effective optimization and numerical solutions for parameters estimation. We obtained appreciable robustness using LevenbergMarquardt approach with sensitivity equations. The proposed methodology has proved effective, at least for structures with two and three compartments. The methodology has also been applied to a small set of actual exams, with results within the expected range, but without more objective assessment due to the absence of a gold standard. 


\section{Introdução}

A técnica de tomografia por emissão de pósitrons, conhecida no meio médico pela sigla "PET" (Positron Emission Tomography), é empregada no estudo não invasivo do corpo humano. Com o auxílio de processamento de imagens médicas, este método permite investigar a integridade e o mecanismo metabólico de órgãos como o cérebro e o coração, possibilitando dessa maneira o diagnóstico precoce de patologias e planejamentos cirúrgicos. As informações fisiológicas reveladas através de um estudo dessa categoria de tomografia são de grande utilidade para atividades clínicas e práticas científicas, apresentando aplicabilidade notável nas áreas de oncologia, neurologia e cardiologia (Carson, 2005; Cobelli, 2000).

Baseado na variação ao longo do tempo da concentração de uma substância marcadora (também conhecida como radiofármaco ou tracer), o método de tomografia por emissão de pósitrons emprega os conhecidos modelos compartimentais no estudo dinâmico do metabolismo da glicose em certas regiões do miocárdio. A análise de viabilidade do miocárdio e a detecção de doenças cardíacas podem ser realizadas baseando-se na estimação de parâmetros característicos da dinâmica via modelagem matemática (Carson, 2005).

No entanto, a escolha do modelo mais adequado não é trivial. Se o modelo escolhido for muito simples, detalhes da dinâmica não serão observados, enquanto que se o modelo for muito complexo (vários compartimentos), a estimativa dos parâmetros torna-se muito imprecisa.

O principal objetivo deste trabalho é uma abordagem para determinar um modelo compartimental para um dado exame, baseado em critérios objetivos. Esta investigação também envolve formas efetivas da estimação de parâmetros da dinâmica fisiológica, bem como a avaliação de todo o processo. Para tal finalidade, utilizaram-se dados sintetizados levando em conta as principais características de exames reais e o critério de informação de Akaike (1974).

\section{Materiais e Métodos}

\section{Descrição da técnica PET}

O emprego da técnica de tomografia por emissão de pósitrons com abordagem dinâmica tem- se tornado cada vez mais difundido em aplicações médicas, sendo capaz de informar aos profissionais da área, por exemplo, as taxas metabólicas de determinados órgãos do corpo humano. Sua importância é vasta: o acompanhamento e tomada de decisão sobre formas de tratamento de alguns tumores cancerígenos podem ser realizados em razão da alta taxa metabólica que estas neoplasias malignas costumam apresentar. A viabilidade do miocárdio também pode ser estudada em função das características metabólicas do músculo cardíaco, tendo como alvos principais a detecção de isquemia cardíaca e fibrose muscular (Guyton e Hall, 1997).

As informações de interesse, na realização de um exame de tomografia por emissão de pósitrons em sua categoria dinâmica, são obtidas através da estimação de parâmetros que governam os chamados modelos compartimentais.

Para ilustração e exemplificação da prática desta técnica, o procedimento pode ser sucintamente descrito da seguinte forma (Figura 1): inicialmente, injeta-se uma pequena porção de radiofármaco na artéria radial do indivíduo. As contagens radioativas provenientes do interior de seu corpo são captadas por um tomógrafo que disponibiliza uma série temporal de imagens apresentando a concentração de medicamento em determinada região do corpo do indivíduo em função do tempo (Huang et al., 1980). Tais regiões são delimitadas através do processamento de imagens médicas e denominadas Regions of Interest (ROIs).

Para avaliar o funcionamento e a robustez da abordagem proposta, utilizamos dados sintetizados reunindo características de exames reais. Para tanto, depois de fixada uma função de entrada exponencial e para cada um dos três modelos compartimentais considerados, foram simulados trinta experimentos com componente aleatória do tipo Poisson. A natureza desse tipo de ruído é sugerida por (Carson, 2005) e característica em medições envolvendo emissões radioativas como é o caso da técnica PET. Repetiu-se o procedimento considerando os outros dois modelos compartimentais restantes, resultando em um conjunto com um total de noventa curvas sintetizadas.

\section{Modelagem matemática}

Tradicionalmente empregados, desde o início da técnica de tomografia por emissão de pósitrons (Huang et al., 1980), modelos compartimentais constituem uma abordagem clássica na estimação de taxas metabólicas. Em PET, cada tecido, órgão (ou cada região deste) é tratado como um compartimento. Dessa maneira, para um estudo cardíaco, por exemplo, pode-se estudar o mecanismo fisiológico do miocárdio utilizando-se três compartimentos: um representando a concentração de radiofármaco no plasma sanguíneo, outro para a concentração de substâncias no meio intersticial muscular e um terceiro para a concentração de substâncias nas células musculares. Cada interligação entre compartimentos é caracterizada por uma constante 


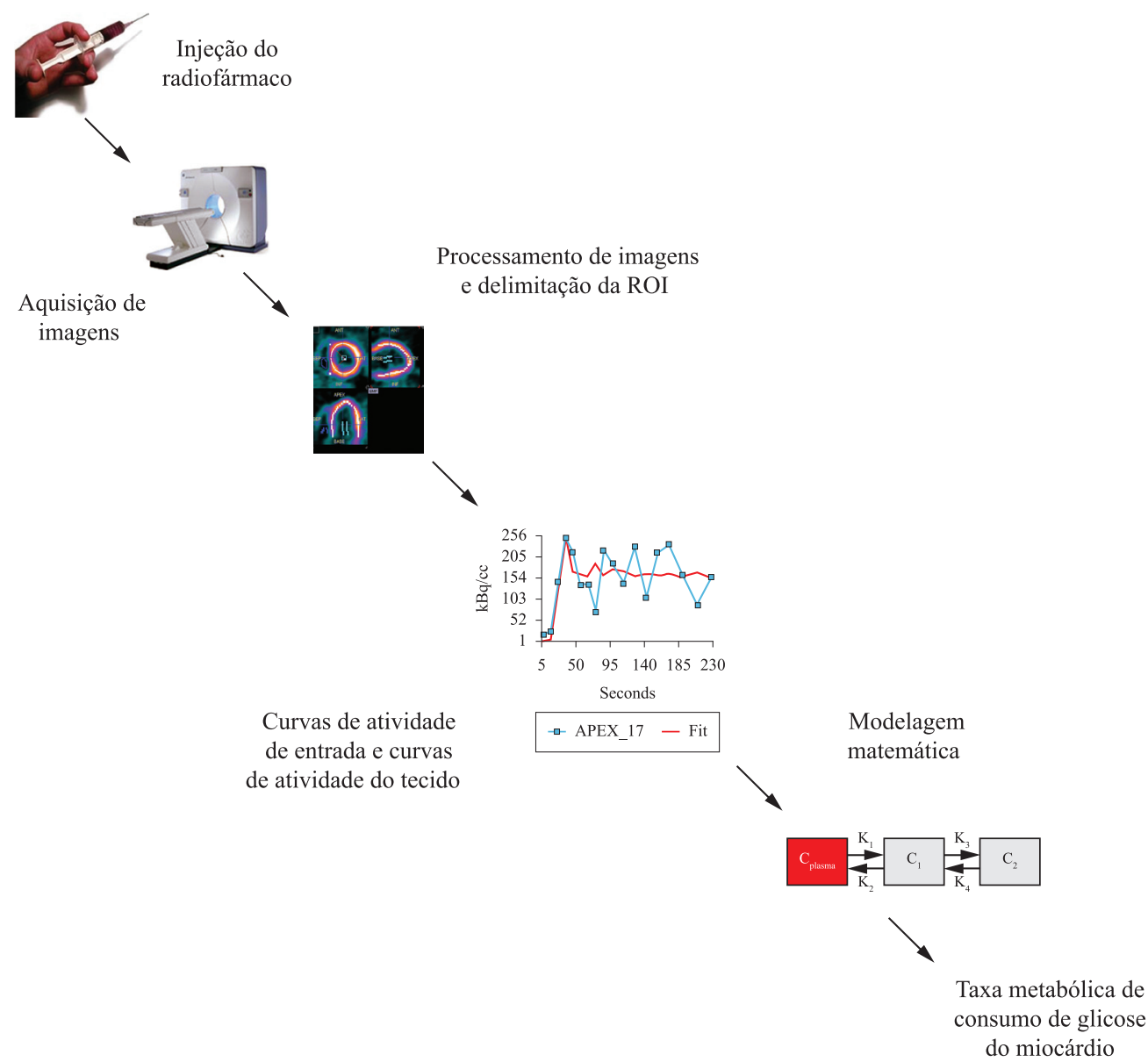

Figura 1. Esquema do procedimento: o radiofármaco é injetado no paciente; as imagens são obtidas através do tomógrafo; regiões de interesse (ROI) são definidas por processamento de imagens; as curvas de entrada podem ser obtidas por amostragem sanguínea ou processamento de imagens; as curvas de atividades são obtidas por processamento de imagens; estimativa dos parâmetros.

Figure 1. Illustration of a typical procedure: initially, injection of a tracer; radioactive counts and reconstruction; the regions of interest (ROI) are delimited by image processing; input data can be obtained by arterial sampling or image processing; time activity curves; parameters estimation.

cinética que revela a taxa com que o radiofármaco e suas variáveis resultantes de metabolização são trocados entre si.

Em caráter informativo, ressalta-se a existência de outras técnicas para modelagem de sistemas biológicos, destacando-se a modelagem não-compartimental e a modelagem a parâmetros distribuídos. A modelagem não-compartimental permite a obtenção de estimativa de parâmetros fisiológicos como o tempo médio de metabolização de determinadas substâncias em função da área sob a curva temporal de concentração de substância marcadora no tecido que se deseja investigar. No entanto, em razão de algumas simplificações com relação ao encaminhamento e metabolismo das moléculas de radiofármaco, muitas vezes não é capaz de descrever tão completa e detalhadamente o organismo em questão quanto a modelagem compartimental (Carson, 2005; Riviere, 1999).
Por outro lado, os modelos a parâmetros distribuídos excedem os modelos compartimentais em termos de detalhamento: tal modelagem preocupa-se não somente em descrever a velocidade com que as substâncias são intercambiadas entre regiões ou órgãos de interesse, mas também de que maneira essa grandeza é influenciada por mecanismos como capilaridade e difusão, por gradiente de concentrações, característicos. Dessa maneira, em termos de complexidade de modelos, diz-se que a modelagem compartimental permanece entre as duas anteriormente descritas.

A Modelagem Matemática compartimental apresentada baseia-se na aplicação do conceito de balanço de massa a cada um dos compartimentos, resultando em um sistema de equações diferenciais ordinárias com função de entrada. Neste artigo, são consideradas três estruturas compartimentais que possuem dois, três e quatro compartimentos. No entanto, 
a abordagem pode considerar um número arbitrário de compartimentos. Os modelos e as respectivas equações (Carson, 2005) são os seguintes (Figura 2):

Equação para modelo com dois compartimentos (A):

$$
\frac{d C_{1}(t)}{d t}=K_{1} \cdot C_{p}(t)-k_{2} \cdot C_{1}(t)
$$
(B):

Equações para modelo com três compartimentos

$$
\begin{aligned}
& \frac{d C_{1}(t)}{d t}=K_{1} \cdot C_{p}(t)+k_{4} \cdot C_{2}(t)-k_{2} \cdot C_{1}(t)-k_{3} \cdot C_{1}(t) \\
& \frac{d C_{2}(t)}{d t}=k_{3} \cdot C_{1}(t)-k_{4} \cdot C_{2}(t)
\end{aligned}
$$

Equações para modelo com quatro compartimentos (C):

$$
\begin{aligned}
\frac{d C_{1}(t)}{d t} & =K_{1} \cdot C_{p}(t)+k_{4} \cdot C_{2}(t)+k_{6} \cdot C_{3}(t) \\
& -\left(k_{2}+k_{3}+k_{5}\right) \cdot C_{1}(t)
\end{aligned}
$$

$\frac{d C_{2}(t)}{d t}=k_{3} \cdot C_{1}(t)-k_{4} \cdot C_{2}(t)$

$\frac{d C_{3}(t)}{d t}=k_{5} \cdot C_{1}(t)-k_{6} \cdot C_{3}(t)$

Com relação às grandezas de interesse, $C_{\mathrm{i}}$ $(i=1,2,3, \ldots)$ representa a concentração de substância marcadora do compartimento. No contexto do estudo, o meio externo é considerado um compartimento adicional contendo o plasma sanguíneo do indivíduo, e a sua concentração $\left(C_{\mathrm{p}}\right)$ é a curva de atividade de entrada. As constantes relativas aos fluxos de material são representadas na Figura 2.

Cada fluxo é diretamente proporcional à concentração de seu compartimento de origem $\left(C_{\mathrm{i}}(t)\right)$, caracterizado por uma constante cinética k. Admite-se que tal proporcionalidade seja invariante no tempo. A constante $\mathrm{K}_{1}$ grafada em maiúsculo por possuir unidade de medida diferente do restante das outras constantes. A Função de Entrada $C_{\mathrm{p}}$ representa a função de entrada dos três sistemas de equações diferenciais e, para estudos cardíacos, representa a concentração do radiofármaco presente no plasma sanguíneo do indivíduo. Esta informação pode também ser obtida por processamento de imagens como visto em van der Weerdt et al. (2001).

As Equações 1-6 podem ser integradas numericamente na plataforma Matlab $^{\circledR}$ empregando-se o método de Runge-Kutta, p.ex. ode45, dispensando sua solução analítica. Todas as condições iniciais são assumidas nulas.

\section{Ajuste de dados}

Obtidas as medidas do tomógrafo, faz-se necessária a aplicação dos modelos compartimentais apresentados com a finalidade de se estimar um conjunto de parâmetros fisiológicos representados pelas constantes características de cada uma das três estruturas consideradas. Cabe aqui uma observação com relação aos dados medidos e dados previstos pelos modelos: em razão do processo de metabolismo do radiofármaco ocorrer em nível molecular, é impossível o tomógrafo distinguir que contagem radioativa proveio de um ou de outro compartimento ou tecido. Portanto, o que se afere com esta técnica de tomografia é a somatória das atividades para cada compartimento.

A função de saída dada pela somatória de concentrações individuais de todos os compartimentos é um importante conceito a ser empregado no Ajuste de Dados, pois dele depende a seguinte função custo de ajuste:
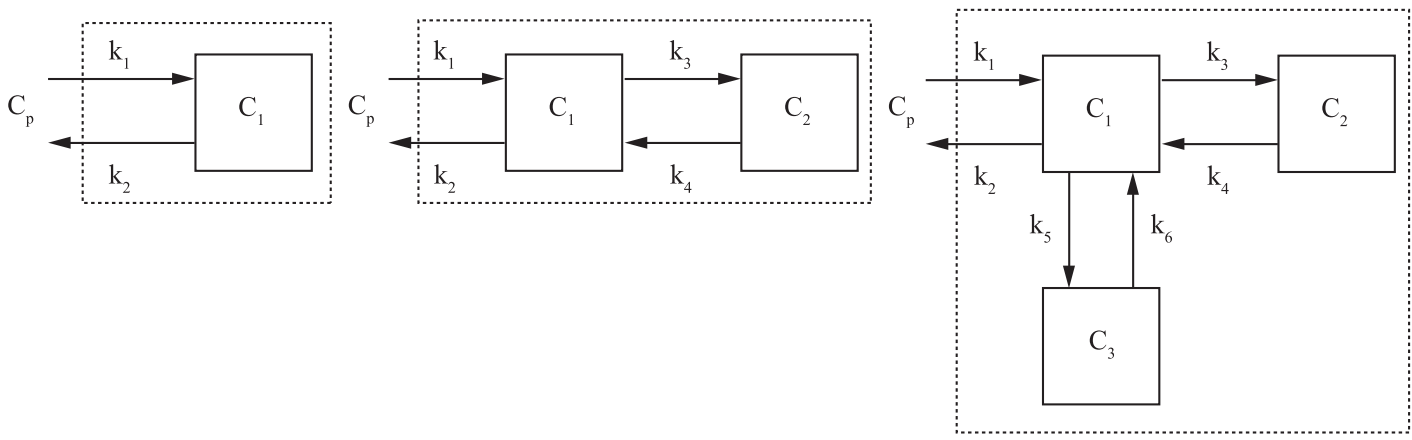

Figura 2. a) Estrutura com dois compartimentos, b) estrutura com três compartimentos e c) estrutura com quatro compartimentos. Figure 2. a) Models with two compartments, b) three compartments and c) four compartments. 
$\chi^{2}=\sum_{i=1}^{n} w_{i} \cdot\left[C_{T A C}\left(t_{i}\right)-\hat{C}_{T A C}\left(t_{i}\right)\right]^{2}$

Tal que:

$\chi^{2}$ é a somatória ponderada dos resíduos quadráticos.

$n$ é o número de dados aferidos.

$\hat{C}_{T A C}\left(t_{i}\right)$ é o valor de concentração previsto pelo modelo no instante de tempo $i$. É dado pela somatória das concentrações individuais de todos os compartimentos, por exemplo, no caso de três compartimentos, $\hat{C}_{T A C}\left(t_{i}\right)=\widehat{C}_{1}\left(t_{i}\right)+\hat{C}_{2}\left(t_{i}\right)$. Tal que:

$C_{\mathrm{TAC}}\left(t_{\mathrm{i}}\right)$ é o valor de concentração aferido no instante de tempo $i$.

$w_{\mathrm{i}}$ é o fator de ponderação dos resíduos quadráticos.O fator de ponderação $w_{\mathrm{i}}$ é tomado como sendo o inverso da variância e, no caso da distribuição dos erros apresentar característica da distribuição de Poisson, é dado pelo inverso da medida aferida no instante $i$ (Carson, 2005).

$w_{i}=\frac{1}{\operatorname{var}\left(C_{T A C}\left(t_{i}\right)\right)}=\frac{1}{C_{T A C}\left(t_{i}\right)}$

Para estimar o conjunto de parâmetros fisiológicos, resolve-se o problema de minimização da função custo da Equação 7. A abordagem escolhida para tal tarefa é o emprego do método de Levenberg-Marquardt (Bard, 1974; Chapras e Canalle, 2008), comumente utilizado para a tarefa de ajuste de dados a modelos não-lineares em seus parâmetros e de natureza iterativa.

A escolha deste método deve-se principalmente ao seu bom desempenho quando comparado a outros métodos de otimização (Bard, 1974). Além disso, o método também possibilita a estimativa de um limite inferior para incertezas das estimativas encontradas através do procedimento de minimização da função custo da Equação 7 (Cobelli et al., 2000).

No entanto, o método de Levenberg-Marquardt requer informações adicionais sobre a função custo, como suas derivadas de primeira e segunda ordem. Tais requisitos são atendidos utilizando-se as chamadas equações de sensibilidade (Bard, 1974; Orsini, 1985; Pronzato e Walter, 1997), as quais fornecem as derivadas parciais da função custo em relação a cada uma das constantes que se deseja estimar e conferem desempenho considerável no procedimento de otimização. As equações de sensibilidade resultam da derivação parcial em relação a cada parâmetro característico das Equações 1-6, conduzindo a um novo conjunto de equações diferenciais. Elas devem ser integradas conjuntamente com as equações diferenciais originais dos modelos compartimentais e também possuem condições iniciais nulas.

\section{Aplicação do método de Levenberg-Marquardt na estimativa dos parâmetros}

Minimizando-se a função custo (Equação 7) em relação ao vetor de parâmetros (constantes $k$ representadas pelo vetor $p$ ), e considerando apenas os 2 primeiros termos da série de Taylor da derivada, tem-se (Pronzato e Walter, 1997):

$\Delta p_{q}=-\left(2 \cdot J^{T} \cdot W \cdot J+\lambda \cdot I\right)^{-1} \cdot 2 \cdot J^{T} \cdot W \cdot r$

Onde:

$\Delta p_{\mathrm{q}}$ é o q-ésimo vetor incremental que atualiza o vetor de parâmetros $(p)$ anterior;

$r$ é o vetor de resíduos, com $n$ componentes, sendo $n$ o número de medidas de $\mathrm{C}_{\mathrm{TAC}}$;

$J$ é amatriz jacobiana dos resíduos, ou seja, $J_{i, j}=\frac{\partial r_{i}}{\partial p_{j}}$ representa o i-ésimo componente de um vetor coluna relativo ao j-ésimo parâmetro;

$W$ é a matriz quadrada diagonal de dimensão $n$ com os elementos da diagonal principal correspondentes ao inverso do valor de cada medida $i$, como estabelecido na relação (8);

$I$ é a matriz identidade;

$\lambda$ é o parâmetro escalar característico do algoritmo de Levenberg-Marquardt que permite um comportamento misto entre o algoritmo de GaussNewton e o método do gradiente. Isso confere ao método maior desempenho em problemas de ajuste a modelos de natureza não-linear.A inversão da matriz da expressão dada pela Equação 9 pode, algumas vezes, resultar em instabilidade numérica devida ao seu mau condicionamento, observada principalmente para o modelo com quatro compartimentos. Dessa forma, utilizou-se a rotina de (Shrager et al., 1994), que por realizar uma espécie de escalonamento matricial apropriado, oferece melhores resultados.

A estimação de parâmetros característicos dos modelos compartimentais não é uma tarefa simples. Em algumas ocasiões, o modelo não é unicamente identificável, isto é, a minimização da função custo pode resultar em diferentes valores dos parâmetros (Cobelli et al., 2000). Este seja talvez o maior problema na estimação de parâmetros para modelos não-lineares, uma vez que diferentes inicializações podem resultar em estimativas distintas fornecidas pelo algoritmo. Diz-se que, então, a função custo possui diversos mínimos locais. Cabe ao usuário uma interpretação minuciosa dos resultados obtidos a fim de não cometer um equívoco em um diagnóstico clínico por causa dos resultados fornecidos por esta metodologia. 


\section{Aplicação das equações de sensibilidade na otimização de Levenberg-Marquardt}

O algoritmo de otimização (9) fornece novas estimativas dos parâmetros iterativamente com o objetivo de minimizar a função da somatória dos resíduos quadráticos. A partir de um palpite inicial para os valores destes parâmetros, a rotina de otimização é executada até um critério de parada ser alcançado.

Baseado numa expansão de Taylor da função objetivo, esse algoritmo depende da avaliação da função objetivo e da avaliação sua derivada com respeito a cada um dos parâmetros a ajustar. Algumas vezes, tais derivadas podem ser difíceis de serem obtidas analiticamente em razão da complexidade das equações regentes do modelo. Uma alternativa seria estimar os valores de derivadas numericamente pelo "método das perturbações" ou "método das diferenças finitas". Entretanto, melhores desempenhos foram observados calculando-se as chamadas equações de sensibilidade das equações diferenciais resultantes da aplicação do conceito de balanço de massa (Bard, 1974; Orsini, 1985; Pronzato e Walter, 1997).

Neste caso, cada equação diferencial correspondente à variação de concentração de tracer é diferenciada com respeito a cada um dos parâmetros a ajustar, e integradas numericamente em conjunto com as equações diferenciais do modelo. Como exemplo, considere um modelo com três compartimentos e quatro parâmetros a estimar. O número total de equações diferenciais a serem integradas pelo método de Runge-Kutta é dez: as duas equações originais do modelo mais as oito equações diferenciais resultantes da diferenciação dessas duas equações originais com respeito a cada um de seus quatro parâmetros característicos $\mathrm{K}_{1}, \mathrm{k}_{2}, \mathrm{k}_{3} \mathrm{e} \mathrm{k}_{4}$.

$\mathrm{O}$ que se deseja destacar neste ponto é que as derivadas necessárias pelo algoritmo de otimização podem ser obtidas diretamente de um método de integração numérica como Runge-Kutta, por exemplo.

A seguir, detalharemos a obtenção das equações de sensibilidade para modelo de dois compartimentos:

Equação do modelo:

$\frac{d C_{1}(t)}{d t}=K_{1} \cdot C_{p}(t)-k_{2} \cdot C_{1}(t)$

Parâmetros em questão: $\mathrm{K}_{1}$ e $\mathrm{k}_{2}$.

Com dois parâmetros em questão, têm-se duas equações de sensibilidade.

Derivando-se (10) com relação à $\mathrm{K}_{1}$ :

$\frac{\partial}{\partial t}\left[\frac{d C_{1}(t)}{d K_{1}}\right]=C_{p}(t)-k_{2} \cdot\left[\frac{d C_{1}(t)}{d K_{1}}\right]$

Derivando-se (10) com relação à $\mathrm{k}_{2}$ : $\frac{\partial}{\partial t}\left[\frac{d C_{1}(t)}{d k_{2}}\right]=-C_{1}(t)-k_{2} \cdot\left[\frac{d C_{1}(t)}{d k_{2}}\right]$

Note que foi utilizada a regra da cadeia e o diferencial com respeito ao tempo e o diferencial com respeito às constantes foram trocados de ordem: ( $\partial t$ e $\left.d K_{1}\right)$, por exemplo. Isso permite que as Equações 10-12 sejam integradas conjuntamente com respeito ao tempo e a matriz Jacobiana pode ser formada, por colunas, da seguinte maneira:

$J=-\left[f\left(K_{1}\right) \quad \vdots f\left(k_{2}\right)\right]$

Onde:

$f\left(K_{1}\right)$ é o vetor coluna que possui os valores resultantes da integração numérica da equação de sensibilidade (11) ao longo do tempo.

$f\left(K_{2}\right)$ é o vetor coluna que possui os valores resultantes da integração numérica da equação de sensibilidade (12) ao longo do tempo.

Se determinado exame possuir, por exemplo, 60 pontos medidos, a matriz Jacobiana $J$ possuirá duas colunas (em razão de se ter duas constantes em questão) e 60 linhas (em razão da integração numérica das Equações 11 e 12, avaliada em todos os respectivos instantes e tempo dos valores medidos). As condições iniciais destas equações de sensibilidade também são nulas.

Esquematicamente, tem-se a (Figura 3):

A aplicação do mesmo raciocínio conduz às equações de sensibilidade para modelos com três e quatro compartimentos (Maeda, 2010). Note-se que para o cálculo da matriz Jacobiana, não foi necessário derivar numericamente em relação aos parâmetros.

\section{Seleção de modelos e avaliação do método}

Para avaliar o funcionamento e robustez do método de Levenberg-Marquardt utilizando equações de sensibilidade propôs-se utilizar dados sintetizados reunindo características de exames reais. Para tanto, depois de fixada uma função de entrada exponencial e escolhido um dos três modelos compartimentais com suas respectivas constantes do escopo deste trabalho, foram geradas trinta curvas de dados adicionadas a realizações de componente aleatória do tipo Poisson. A natureza desse tipo de ruído é sugerida por (Carson, 2005) e característica em medições envolvendo emissões radioativas como é o caso da técnica PET.

Na obtenção de dados sintetizados, foram levadas em consideração as ordens de grandeza dos valores absolutos de constantes dos modelos compartimentais e a intensidade da componente aleatória do tipo Poisson encontradas na prática. Para caracterização 


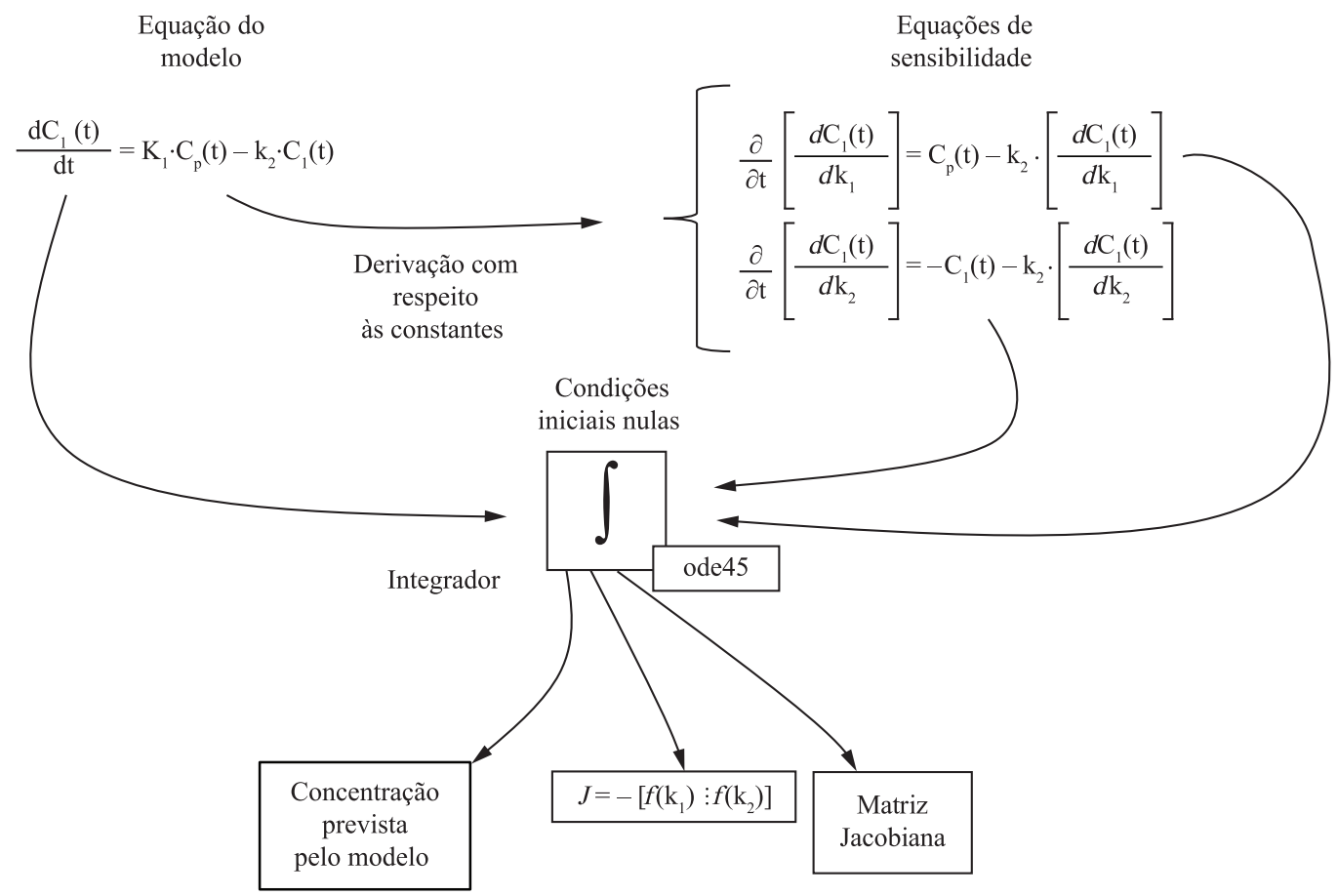

Figura 3. Esquema para integração das equações de sensibilidade e obtenção da matriz Jacobiana.

Figure 3. Illustration of the integration of sensitivity equations and Jacobian matrix.

do ruído inerente à amostragem da concentração de substância marcadora nos tecidos e, permitir a síntese de dados, foram utilizados resultados obtidos após ajuste de curvas de casos reais por software comercial. Dessa maneira, estimou-se a ordem de grandeza do valor da variância do ruído e utilizou-se gerador de números randômicos com distribuição Poisson para realizações deste processo aleatório.

Repetiu-se o procedimento considerando os outros dois modelos compartimentais restantes, resultando em um conjunto com um total de noventa curvas sintetizadas. O método de estimação de parâmetros foi então aplicado utilizando-se as três estruturas compartimentais apresentadas para cada uma das noventa curvas sintetizadas, na esperança de que houvesse concordância entre o modelo e respectivas constantes utilizadas na síntese de dados com o modelo e respectivas constantes encontradas após a tarefa de estimação de parâmetros.

O modelo mais adequado foi selecionado utilizando-se o critério de informação de Akaike (AIC) (Akaike, 1974), dado pela seguinte expressão:

$A I C=n \cdot \ln \left(\chi^{2}\right)+2 \cdot K$

Onde:

$\chi^{2}$ é a somatória ponderada dos resíduos quadráticos. $n$ é o número de dados aferidos.

$K$ é o número de constantes fisiológicas do modelo.

Dessa maneira, é possível selecionar o modelo compartimental mais adequado quando do ajuste de um exame sintético empregando-se todas as três estruturas compartimentais consideradas. Para isso, após a tarefa de estimação para uma curva sintetizada, compararam-se os três índices AIC obtidos e escolheu-se o modelo com menor valor desta métrica. Se o modelo escolhido através do índice de Akaike coincidisse com o modelo utilizado na síntese de dados, um acerto era computado a favor da metodologia. Caso contrário, nenhum ponto era computado. O critério de Akaike procura estabelecer um compromisso entre a quantidade de parâmetros característicos do modelo e o valor de erro quadrático ponderado atingido após a estimação dos parâmetros, indicando falta ou excesso de ajuste dos dados.

Repetiu-se o procedimento das noventa curvas com três níveis de intensidade de ruído Poisson: nível médio, obtido de exames reais; nível baixo, com valor de concentração de radiofármaco dez vezes maior do que o utilizado para nível médio e nível alto, com valor de concentração de radiofármaco dez vezes menor do que o utilizado para nível médio. A escolha é justificada em razão da característica de ruído tipo Poisson, o qual apresenta menor potência 


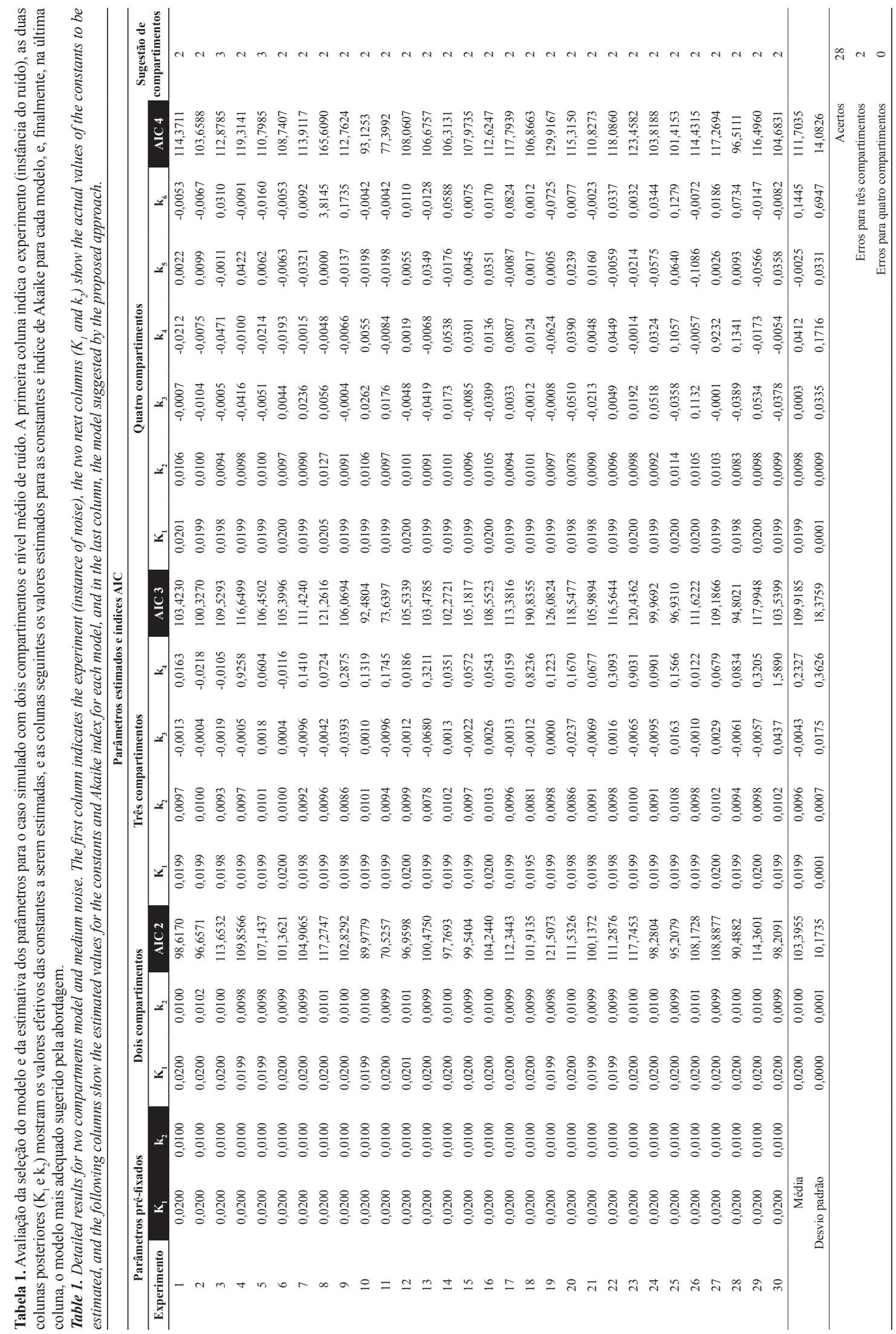


relativa para maiores valores de concentração no tecido humano e vice-versa. Para o ruído médio, adotou-se o valor médio de concentração temporal de radiofármaco de $20000 \mathrm{kBq} / \mathrm{mL}$, número este baseado em exames reais.

\section{Resultados}

Para cada nível de ruído (baixo, médio e alto) e para cada modelo (dois, três e quatro compartimentos), foi avaliada a capacidade da abordagem proposta de indicar corretamente o modelo mais adequado, bem como o erro da estimativa dos parâmetros. Portanto, foram avaliadas nove configurações distintas.

A Tabela 1 exemplifica os resultados para a configuração com modelo de dois compartimentos e ruído médio. As demais estão em (Maeda, 2010). A primeira coluna indica o experimento (instância do ruído), as duas colunas posteriores $\left(\mathrm{K}_{1}\right.$ e $\left.\mathrm{k}_{2}\right)$ mostram os valores efetivos das constantes a serem estimadas, e as colunas seguintes os valores estimados para as constantes e índice de Akaike para cada modelo, e finalmente na última coluna, o modelo mais adequado sugerido pela abordagem em função do AIC.

Os números de acertos observados na identificação de parâmetros dos três grupos de trinta experimentos sintetizados são apresentados nas Tabelas 2-4 (dois, três e quatro compartimentos), para os níveis de ruído baixo, médio e alto, respectivamente. $\mathrm{O}$ objetivo desta abordagem é verificar a robustez da metodologia proposta, tanto em relação aos diferentes níveis de ruído quanto em relação a diferentes inicializações dos valores das constantes a serem determinadas.

Com o objetivo de avaliar os graus de concordância entre os modelos empregados para síntese de dados e os modelos identificados após a estimação paramétrica,

Tabela 2. Matriz de confusão, com nível de ruído baixo, para os três modelos avaliados, cada qual com trinta experimentos.

Table 2. Confusion matrix, with low level noise, for the three investigated models. Each model assessed by thirty experiments.

\begin{tabular}{|c|c|c|c|c|c|c|}
\hline \multicolumn{7}{|c|}{ Proposto pelo decisor de modelos } \\
\hline & Ruído baixo & $\begin{array}{c}\text { Dois } \\
\text { compartimentos }\end{array}$ & $\begin{array}{c}\text { Três } \\
\text { compartimentos }\end{array}$ & $\begin{array}{c}\text { Quatro } \\
\text { compartimentos }\end{array}$ & $\begin{array}{l}\text { Totais das } \\
\text { linhas }\end{array}$ & $\begin{array}{c}\text { Frequência } \\
\text { esperada }\end{array}$ \\
\hline \multirow{11}{*}{ } & Dois compartimentos & 27 & 2 & 1 & 30 & 9,0000 \\
\hline & Três compartimentos & 0 & 30 & 0 & 30 & 10,6667 \\
\hline & Quatro compartimentos & 0 & 0 & 30 & 30 & 10,3333 \\
\hline & Totais das colunas & 27 & 32 & 31 & 90 & 30,0000 \\
\hline & Total da diagonal & 87 & & & & \\
\hline & Total da diagonal (\%) & $96,67 \%$ & & & & \\
\hline & Índice Kappa & 0,9500 & & & & \\
\hline & Valor-p & $<0,001$ & & & & \\
\hline & Intervalo de confiança cc & n $95 \%$ & & & & \\
\hline & Inferior & 0,8040 & & & & \\
\hline & Superior & 1,0000 & & & & \\
\hline
\end{tabular}

Tabela 3. Matriz de confusão, com nível de ruído médio, para os três modelos avaliados, cada qual com trinta experimentos.

Table 3. Confusion matrix, with medium level noise, for the three investigated models. Each model assessed by thirty experiments.

\begin{tabular}{|c|c|c|c|c|c|c|}
\hline \multicolumn{7}{|c|}{ Proposto pelo decisor de modelos } \\
\hline & Ruído médio & $\begin{array}{c}\text { Dois } \\
\text { compartimentos }\end{array}$ & $\begin{array}{c}\text { Três } \\
\text { compartimentos }\end{array}$ & $\begin{array}{c}\text { Quatro } \\
\text { compartimentos }\end{array}$ & $\begin{array}{c}\text { Totais das } \\
\text { linhas }\end{array}$ & $\begin{array}{c}\text { Frequência } \\
\text { esperada }\end{array}$ \\
\hline \multirow{11}{*}{ } & Dois compartimentos & 28 & 2 & 0 & 30 & 9,3333 \\
\hline & Três compartimentos & 0 & 29 & 1 & 30 & 10,6667 \\
\hline & Quatro compartimentos & 0 & 1 & 29 & 30 & 10,0000 \\
\hline & Totais das colunas & 28 & 32 & 30 & 90 & 30,0000 \\
\hline & Total da diagonal & 86 & & & & \\
\hline & Total da diagonal (\%) & $95,56 \%$ & & & & \\
\hline & Índice Kappa & 0,9333 & & & & \\
\hline & Valor-p & $<0,001$ & & & & \\
\hline & Intervalo de confiança cc & n $95 \%$ & & & & \\
\hline & Inferior & 0,7870 & & & & \\
\hline & Superior & 1,0000 & & & & \\
\hline
\end{tabular}


Tabela 4. Matriz de confusão, com nível de ruído alto, para os três modelos avaliados, cada qual com trinta experimentos. Table 4. Confusion matrix, with high level noise, for the three investigated models. Each model assessed by thirty experiments.

\begin{tabular}{|c|c|c|c|c|c|c|}
\hline \multicolumn{7}{|c|}{ Proposto pelo decisor de modelos } \\
\hline & Ruído alto & $\begin{array}{c}\text { Dois } \\
\text { compartimentos }\end{array}$ & $\begin{array}{c}\text { Três } \\
\text { compartimentos }\end{array}$ & $\begin{array}{c}\text { Quatro } \\
\text { compartimentos }\end{array}$ & $\begin{array}{c}\text { Totais das } \\
\text { linhas }\end{array}$ & $\begin{array}{c}\text { Frequência } \\
\text { esperada }\end{array}$ \\
\hline \multirow{11}{*}{ 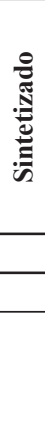 } & Dois compartimentos & 28 & 1 & 1 & 30 & 9,3333 \\
\hline & Três compartimentos & 0 & 29 & 1 & 30 & 16,3333 \\
\hline & Quatro compartimentos & 0 & 19 & 11 & 30 & 4,3333 \\
\hline & Totais das colunas & 28 & 49 & 13 & 90 & 30,0000 \\
\hline & Total da diagonal & 68 & & & & \\
\hline & Total da diagonal $(\%)$ & $75,56 \%$ & & & & \\
\hline & Índice Kappa & 0,6333 & & & & \\
\hline & Valor-p & $<0,001$ & & & & \\
\hline & Intervalo de confiança c & m 95\% & & & & \\
\hline & Inferior & 0,4960 & & & & \\
\hline & Superior & 0,7700 & & & & \\
\hline
\end{tabular}

Tabela 5. Graus de concordância do índice de Kappa. Table 5. Kappa index degrees of aggreement

\begin{tabular}{cc}
\hline Índice de Kappa & Grau de concordância \\
\hline$<0$ & Pobre \\
0,00 a 0,20 & Fraco \\
0,21 a 0,40 & Razoável \\
0,41 a 0,60 & Moderado \\
0,61 a 0,80 & Considerável \\
0,81 a 1,00 & Forte \\
\hline
\end{tabular}

para os três diferentes níveis de ruído, empregou-se o índice de classificação de Kappa (Landis e Koch, 1977), que se encontram listados na Tabela 5.

Os valores de índice da Kappa obtidos para a Tabela 1-3 foram, respectivamente, 0,9500; 0,9333 e 0,6333 .

\section{Discussão e Conclusão}

Como principal resultado deste trabalho, obteve-se a seleção de modelos mediante o emprego do critério de informação de Akaike, além da comprovação da eficácia da metodologia de equações de sensibilidade para a estimação paramétrica de modelos compartimentais caracterizados por equações diferenciais. Foram explorados todas as fases relevantes da análise compartimental, em especial a otimização e soluções numéricas efetivas. Conclui-se que tal abordagem pode vir a ser bastante útil na identificação de outros tipos de sistemas dinâmicos com excitação de entrada, oferecendo até mesmo uma maneira de se estimar limites inferiores para as incertezas dos parâmetros. Comprovou-se também apreciável robustez oferecida pelo método de otimização de Levenberg-
Marquardt, fato este observado pelos elevados índices de concordância de Kappa obtidos neste trabalho. A metodologia revela-se eficaz, ao menos para as estruturas com dois e com três compartimentos.

Adicionalmente, uma interface gráfica foi programada em Matlab ${ }^{\circledR}$ para facilitar a conferência das afirmações descritas neste texto. As rotinas programadas foram elaboradas de maneira modular, facilitando a inclusão de novos modelos para trabalhos futuros. A incorporação do recurso de restrição foi implementada na interface gráfica programada em Matlab $^{\circledR}$, porém de maneira relativamente simples.

Com relação ao ruído incluído nos dados sintetizados, foi utilizada a distribuição do tipo Poisson, conferindo maior proximidade às condições encontradas em exames clínicos. Além disso, observouse grau de concordância do tipo "forte" para níveis de ruído de intensidade baixo $(0.9500)$ e médio $(0,9333)$, indicando que a estimação de parâmetros do modelo mais complexo (com quatro compartimentos), na situação de "Ruído Alto", fica limitada justamente pela maior intensidade deste.

A metodologia aqui descrita também foi aplicada a um pequeno conjunto de exames reais, com resultados dentro da faixa esperada, porém sem avaliação mais objetiva devido à ausência de uma referência padrão (gold standard).

\section{Agradecimentos}

Ao Instituto do Coração do Hospital das Clínicas da Faculdade de Medicina da Universidade de São Paulo - InCor, à FAPESP e ao CNPq. Ao Matheus C. Moraes pelo auxílio na formatação do texto. 


\section{Referências}

Akaike H. A new look at the statistical model identification. IEEE Transactions on Automatic Control. 1974; 19(6):716-23. http://dx.doi.org/10.1109/TAC.1974.1100705

Bard Y. Nonlinear parameter estimation. New York: Academic Press; 1974.

Carson RE. Tracer Kinetic Modeling in PET. In: Bailey D, editor. Positron Emission Tomography - Basic sciences. London: Springer; 2005. p. 127-159. http://dx.doi. org/10.1007/1-84628-007-9_6

Chapras S, Canaller R. Métodos numéricos para engenharia. São Paulo: McGraw Hill; 2008.

Cobelli C, Foster D, Toffolo G. Tracer kinetics in biomedical research - from data to model. New York: Kluwer Academic Publishers; 2000.

Guyton A, Hall J. Tratado de Fisiologia médica. 9th ed. Rio de Janeiro: Guanabara Koogan; 1997.

Huang SC, Phelps ME, Hoffman EJ, Sideris K, Selin CJ, Kuhl DE. Noninvasive determination of local cerebral metabolic rate of glucose in man. The American Physiological Society - Endocrinology and Metabolism. 1980; 238(1):E69-82.
Landis JR, Koch GG. The Measurement of observer agreement for categorical data. Biometrics. 1977; 33(1):159-74. PMid:843571. http://dx.doi.org/10.2307/2529310

Maeda, JEMS. Estimação de parâmetros de modelos compartimentais para Tomografia por Emissão de Pósitrons [tese]. São Paulo: Universidade de São Paulo; 2010.

Orsini LQ. Introdução aos sistemas dinâmicos. Rio de Janeiro: Guanabara Dois; 1985.

Pronzato L, Walter E. Identification of parametric models: from Experimental Data. Paris: Springer; 1997.

Riviere JE. Comparative pharmacokinetics: principles, techniques and applications. Ames: Wiley-Blackwell; 1999.

Shrager R, Jutan A, Muzic R. leasqr.m [Internet]. 1994. [cited 2010 fev. 17]. Available from: http://users.powernet. co.uk/kienzle/octave/matcompat/scripts/optim/leasqr.m.

van der Weerdt AP, Klein LJ, Boellaard R, Visser CA, Visser FC, Lammertsma AA. Image-derived input functions for determination of MRGlu in cardiac 18F-FDG PET scans. The Journal of Nuclear Medicine. 2001; 42(11):1622-29. PMid:11696630

\footnotetext{
Autores 